\title{
Colchicin in der Behandlung der tracheobronchialen Amyloidose
}

\author{
Arturo Morales $^{\mathrm{a}} \quad$ Marizell Pari $^{\mathrm{b}}$ Rosa López-Lisbona ${ }^{\mathrm{b}} \quad$ Noelia Cubero $^{\mathrm{b}} \quad$ Jordi Dorca $^{\mathrm{b}}$ \\ Antoni Rosell ${ }^{\mathrm{b}}$ \\ ${ }^{a}$ Departamento de Enfermedades Respiratorias, Facultad de Medicina, Pontificia Universidad Católica de Chile, Santiago, Chile;

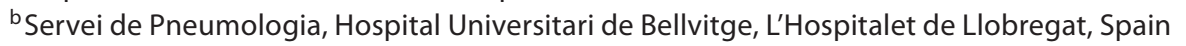

\section{Gesicherte Tatsachen}

- Tracheobronchiale Amyloidose ist eine seltene Krankheit, die mit beträchtlicher Morbidität und Mortalität einhergehen kann.

- Es gibt keine randomisierten Studien, die sich gezielt mit der Behandlung dieser Erkrankung befassen.

\section{Neue Aspekte}

- Colchicin könnte eine wichtige Rolle bei der langfristigen Beherrschung dieser Form der lokalisierten Amyloidose spielen, die anders auf diese Behandlung anspricht als eine systemische Amyloidose.

\footnotetext{
Schlüsselwörter

Tracheobronchiale Amyloidose · Behandlung · Colchicin .

Fallbericht · Kehlkopfamyloidose · Bronchoskopie
}

\section{Zusammenfassung}

Die tracheobronchiale Amyloidose ist eine selten vorkommende Erkrankung, die durch Ablagerungen von Proteinstrukturen im Tracheobronchialbaum gekennzeichnet ist. Die Krankheit ist generell mit einer hohen Morbidität und einer variablen Mortalität in den Jahren nach der Diagnosestellung assoziiert. Über die optimale Behandlung besteht kein Konsens. Wir berichten über den Fall einer63-jährigen Patientin, die sich mit einer diffusen tracheobronchialen Amyloidose mit begleitender Kehlkopferkrankung vorstellte und bei der aufgrund einer hochgradigen subglottischen Stenose eine perkutane Tracheotomie erforderlich wurde; Anzeichen einer systemischen Amyloidose lagen nicht vor. Nach der
Behandlung allein mit Colchicin normalisierte sich der stenosierte Bereich vollständig, mit sehr gutem Ansprechen der tracheobronchialen Amyloidose, von der nur noch kleine gelbe Plaques übrig blieben. In den folgenden 4 Jahren der Nachbeobachtung blieb die Patientin symptomfrei, ohne Anzeichen einer endoskopischen Progression. Dies ist der erste dokumentierte Fall eines solchen Ansprechens auf alleinige Colchicinbehandlung bei tracheobronchialer Amyloidose. Die zum Thema vorliegende Literatur wird im Überblick dargestellt.

(c) 2016 S. Karger GmbH, Freiburg

\section{KARGER}

Fax +497614520714

information@karger.com

www.karger.com

\section{(c) 2016 S. Karger GmbH, Freiburg}

Accessible online at:

www.karger.com/kkp
Antoni Rosell, MD, PhD

Respiratory Endoscopy Unit, Pulmonology Department

IDIBELL, CIBERes, Universitat de Barcelona

Hospital Universitari de Bellvitge, Feixa Llarga s/n

08907 L'Hospitalet de Llobregat, Spanien

arosell@bellvitgehospital.cat 


\section{Einleitung}

Amyloidose ist eine seltene Krankheit, die durch die anomale Ablagerung von Proteinstrukturen im extrazellulären Gewebe gekennzeichnet ist - die Proteine bilden unlösliche Fibrillen mit niedrigem Molekulargewicht, die zu Organschäden führen [1] Die Erkrankung kann systemisch mit ausgedehnter Organbeteiligung oder aber lokal begrenzt sein. Die tracheobronchiale Amyloidose (TBA) ist eine der zahlreichen Manifestationsformen der lokalisierten pulmonalen Amyloidose. Die respiratorische Symptomatik ist unspezifisch; die Diagnose wird anhand des bronchoskopischen Befunds gestellt und anhand der Doppelbrechung von mit Kongorot gefärbtem Gewebe in der Polarisationsmikroskopie gesichert [2]. TBA ist eine nicht häufig vorkommende Erkrankung [3], deren Behandlung in den wenigen veröffentlichten Arbeiten kontrovers diskutiert wird. Wir berichten hier über eine TBA-Patientin mittleren Alters, die hervorragend auf eine Behandlung mit Colchicin ansprach.

\section{Vorstellung des Falls}

Die Patientin ist eine 63-jährige Frau, bei der vor 10 Jahren gestützt auf wiederholte Episoden von Pneumonie in der Vorgeschichte sowie auf positive Kongorot-Färbung und Substanz-P-Nachweis im Bronchiengewebe eine TBA diagnostiziert wurde. Danach war die Patientin symptomfrei, bis in den letzten 2 Monaten vor unserer Erstuntersuchung trockener Husten und Heiserkeit auftraten. In der letzten Woche kamen dann noch eine geringfügige Hämoptyse, Fieber bis zu $38,2{ }^{\circ} \mathrm{C}$ und Asthenie hinzu, jedoch ohne Dyspnoe. Sie zeigte Eupnoe mit einem Pulsoximetriewert von 97\% bei Raumluft. Die körperliche Untersuchung war ohne Befund. Blutbild, Nieren- und Leberfunktion sowie das Harnsediment waren unauffällig. Eine röntgenologische Untersuchung des Thorax ergab eine rechtsseitige perihiläre Verschattung; passend dazu zeigte eine Thorax-Computertomografie (CT) das Bild einer chronischen residuellen, geringfügigen zystischen Bronchiektasie mit muköser Impaktion. Außerdem waren in der CT-Bildgebung regelmäßige konzentrische tracheale und bronchiale Wandverdickungen $\mathrm{zu}$ erkennen (Abb. 1). Eine $\mathrm{zu}$ diesem Zeitpunkt durchgeführte Bronchoskopie zeigte bilateral subkordal verdicktes Gewebe, das eine subglottische Stenose mit 30\% freiem Restlumen bewirkte (Abb. 2a). Die Bestimmung der Durchgängigkeit der subglottischen Stenose erfolgte mittels subjektiver visueller Beurteilung durch einen erfahrenen Bronchoskopen. Die respiratorische Mukosa im Bereich von der Trachea bis zur Carina war mikronodulär, spröde und von entzündlichem Aussehen, ohne signifikante Lumenreduktion - all dies ist mit einer TBA vereinbar (Abb. 3a, b). Die Mikrobiologie ergab negative Mykobakterien- und Myzetenkulturen. Anhand einer negativen Serum- und Urin-Immunfixation, einer unauffälligen Elektrophorese, einer unauffälligen Echokardiografie und einer Fettpolsteraspiration ohne Amyloidablagerungen wurde eine systemische Amyloidose ausgeschlossen. Die quantitative Bestimmung der Immunglobuline ergab ebenfalls normale Werte. Eine Elektromyografie zeigte keine elektrophysiologische Neuropathie. Eine DNAAnalyse auf familiäre Amyloidose war ebenfalls negativ. Die Patientin erhielt eine 10-tägige Kombinationstherapie mit Amoxicillin-Clavulansäure und Kortikosteroiden in einer Dosierung von $1 \mathrm{mg} / \mathrm{kg}$, und als klinische Besserung eintrat, wurde eine Langzeittherapie mit Colchicin $1 \mathrm{mg} / \mathrm{Tag}$ eingeleitet. Einen Monat später suchte die Patientin die Notaufnahme mit Dyspnoe, Fieber und Stridor auf, wobei eine H1N1-Grippe diagnostiziert wurde. Eine antivirale Therapie mit Oseltamivir $75 \mathrm{mg} / \mathrm{Tag}$ wurde eingeleitet. Eine Woche später zeigten sich in einer erneuten Bronchoskopie eine Progression der subglottischen submukösen Verdickung mit 15-20\% verbleibendem freien Tracheallumen sowie Läsionen im rechten Hauptbronchus mit $20 \%$ Stenose. Eine formale Tracheotomie wurde durchgeführt,
Abb. 1. Thorax-CT bei der Erstuntersuchung. Die schwarzen Pfeile weisen auf die ringförmige Wandverdickung in der Luftröhre (a) und im Hauptbronchus (b) hin.
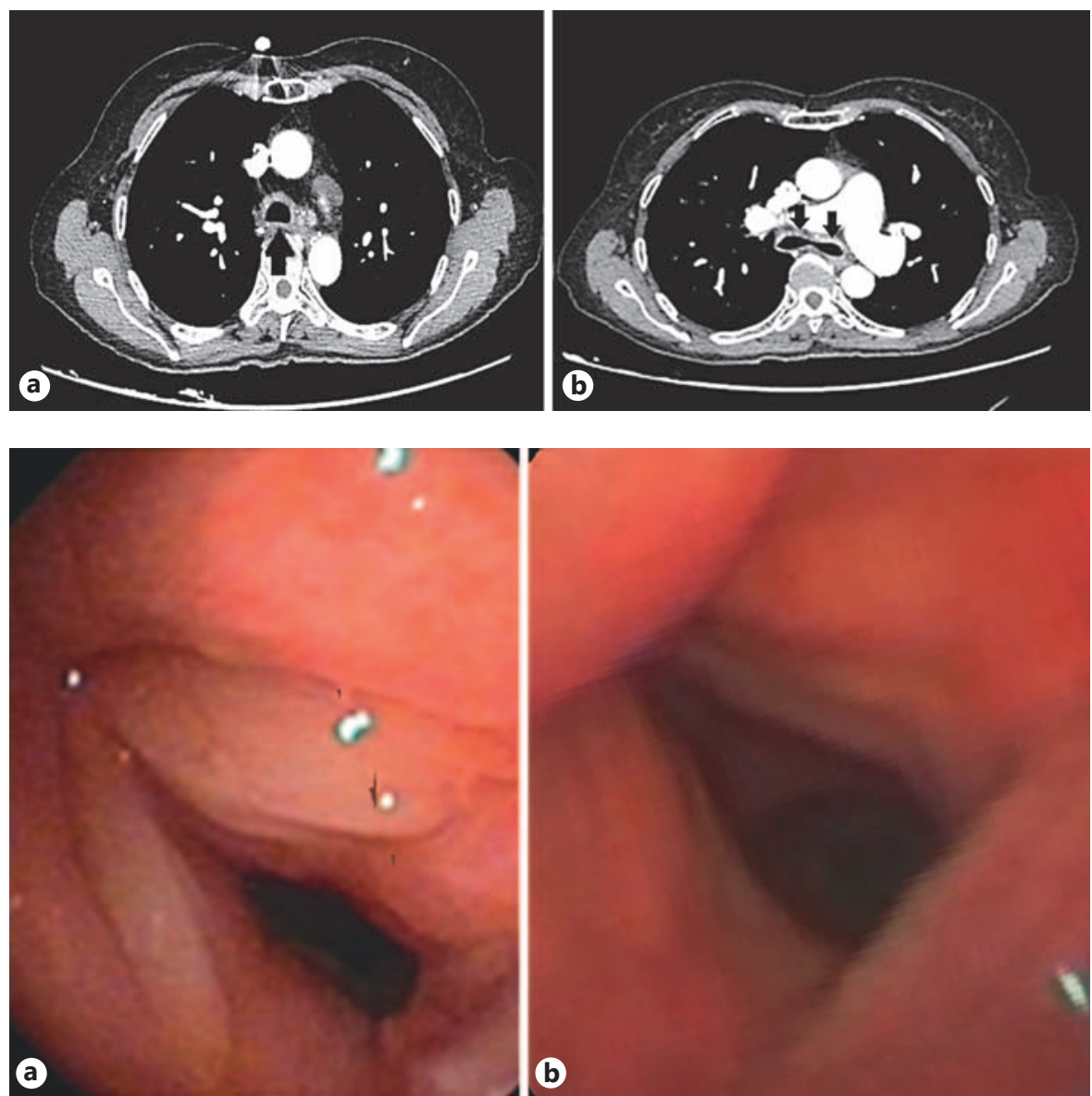

Abb. 2. Bronchoskopische Aufnahmen der subglottischen Stenose (laryngeale Ansicht) bei der Erstuntersuchung (a) und 8 Monate später (b). 

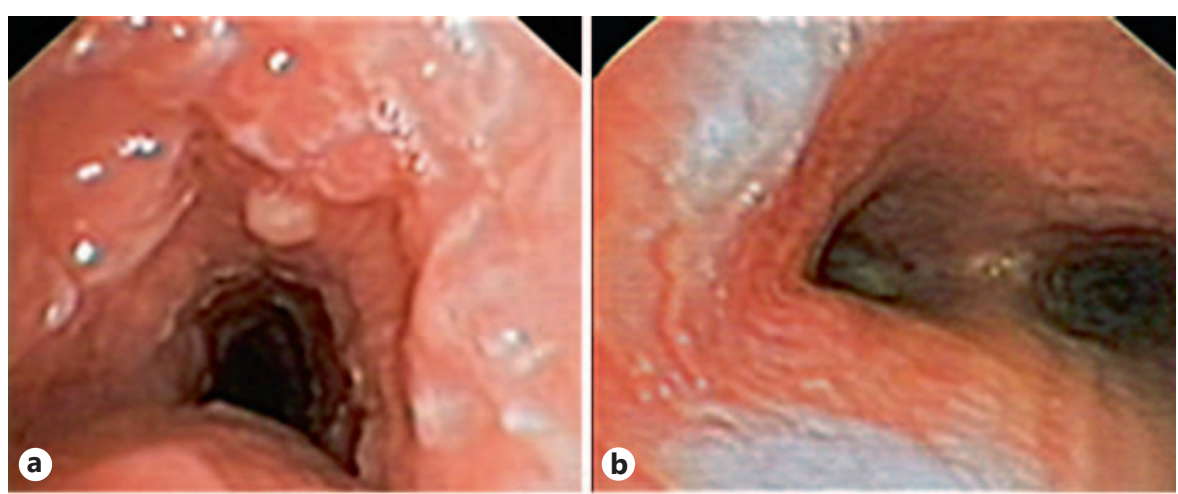

Abb. 3. Tracheobronchiale Erkrankung. Bronchoskopische Bildgebung der Trachea und Carina mit mikronodulär, spröde und entzündlich aussehender Atemwegsschleimhaut von der Trachea bis zur Carina ohne signifikante Lumenreduktion bei der Erstuntersuchung (a b) und mit praktisch normalem Erscheinungsbild nach der Colchicinbehandlung (c, $\mathbf{d})$.
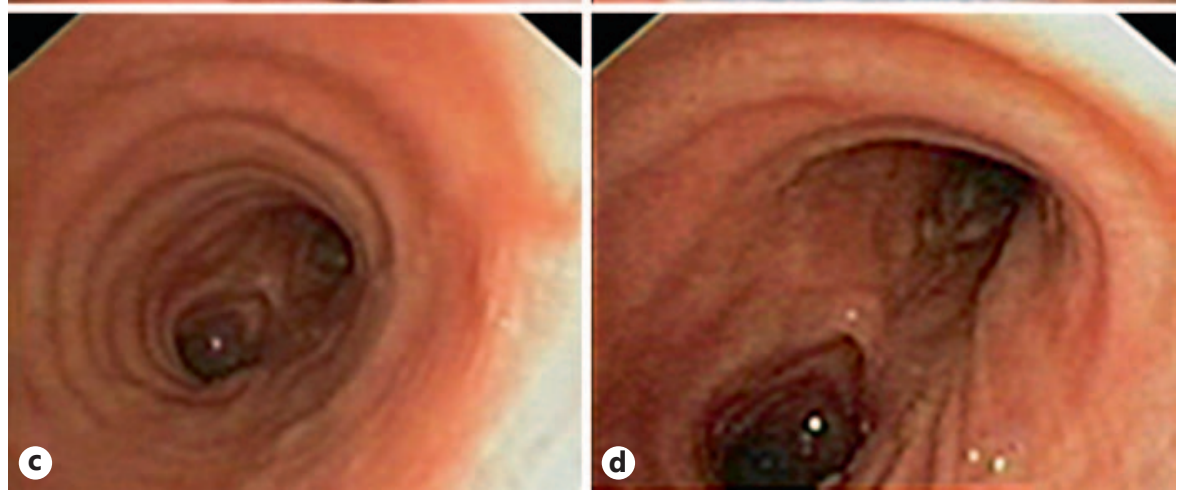

und die Colchicindosis wurde auf 2 mg/Tag erhöht. Nach 4 Monaten Colchicinbehandlung war eine deutliche Besserung der Bildgebung und Symptomatik zu verzeichnen. Weitere Behandlungsmaßnahmen waren nicht erforderlich. 8 Monate später war die Patientin vollständig symptomfrei, die subglottische Stenose war vollständig abgeklungen (Abb. 2b) und der Tracheotomietubus konnte entfernt werden. In einer erneuten flexiblen Bronchoskopie verblieben nur noch kleine gelbe Plaques in der trachealen und bronchialen Mukosa (Abb. 3c, d). Zu diesem Zeitpunkt wurde das Colchicin aufgrund von Alopezie und Leukopenie abgesetzt. In den folgenden 4 Jahren der Nachbeobachtung blieb die Patientin symptomfrei, mit Ausnahme vereinzelter Episoden von Hämoptyse bei Virusinfektionen der Atemwege. Kontrollbronchoskopien ergaben keine Progression.

\section{Diskussion}

TBA ist eine der zahlreichen lokalisierten Manifestationsformen von Amyloidosen der Atemwege. Es liegen Fallberichte von Patienten im Alter von 25-80 Jahren vor, typischerweise wird die Diagnose jedoch um das fünfte Lebensjahrzehnt gestellt. Die größte Fallserie beschreibt eine männliche Prädominanz [4-7]. Die gemeinhin beschriebenen Symptome sind Husten, Dyspnoe, Heiserkeit und gelegentlich Hämoptyse, ähnlich wie in unserem Fall $[1,4,5]$. Den publizierten Fallserien nach zu urteilen, wird die definitive Diagnose in der Regel mit 8-37 Monaten Verzögerung nach dem Einsetzen der Symptome gestellt; häufig wird zunächst fälschlicherweise Asthma oder eine chronisch-obstruktive Lungenerkrankung diagnostiziert $[4,5]$. In der Pathogenese sind die Amyloidfibrillen meist vorwiegend vom AL-Typ, was bestätigt, dass keine systemische monoklonale Leichtkettenkrankheit vorliegt [8]. Einige Berichte vermuten die lokale Produktion amyloidogener leichter Ketten durch sehr kleine lokale Klone von Lymphoplasmazellen $[1,9]$. Wie auch in unserem Fall wird bei Verdacht die Diagnose zunächst anhand des klinischen, radio- logischen und bronchoskopischen Befunds gestellt und dann anhand der Doppelbrechung von Kongorot-gefärbtem Gewebe in der Polarisationsmikroskopie gesichert [2]. Die röntgenologische Untersuchung des Thorax kann bei bis zu 70\% der Patienten unauffällig ausfallen [5], und es liegen nur indirekte Anzeichen für eine Obstruktion mit Atelektase oder Volumenverlust vor [10, 11]. Das Thorax-CT von Patienten mit TBA zeigt typischerweise eine ringförmige tracheobronchiale Verdickung, zum Teil mit Verkalkungen, Lumenverengung, Atelektase und/oder lokalisierter Bronchiektasie $[5,10]$. In der Lungenfunktionsprüfung sind unterschiedliche Muster beschrieben worden, je nach Lokalisation der TBA-Beteiligung [10]. Das bronchoskopische Erscheinungsbild kann entweder durch diffuse submuköse Plaques oder durch lokale tumorähnliche Raumforderungen geprägt sein, wobei Ersteres häufiger beschrieben worden ist [1]. Mittels bronchoskopbasierter konfokaler Endomikroskopie lassen sich In-vivo-Aufnahmen auf Zellebene erstellen, die in von TBA betroffenen Arealen ein «watteähnliches» Erscheinungsbild zeigen [12].

Der klinische Verlauf variiert stark. Während in einigen Fallserien von Mortalitätsraten von bis zu 75\% innerhalb von 6 Jahren ab der Diagnosestellung berichtet wird, verzeichnen andere keinen einzigen Todesfall in einem durchschnittlichen Nachbeobachtungszeitraum von 7,6 Jahren [7]. Die 5-Jahres-Überlebensrate bei der diffusen Form von TBA wird auf $30-50 \%$ geschätzt $[5,13]$. Unsere Patientin blieb bis 10 Jahre nach der Erstdiagnose symptomfrei; eine solche Entwicklung ist auch in anderen Fällen beschrieben worden $[5,14]$. Die tracheobronchiale Progression, die in ihrem Fall zu beobachten war, war mit einer Kehlkopfamyloidose assoziiert. Dies ist insofern nicht überraschend, als zuvor schon von Fällen mit gleichzeitiger laryngealer Amyloidose und 
TBA berichtet worden ist [15], ohne dass Hinweise auf eine zugrunde liegende systemische Amyloidose gefunden wurden.

Es gibt kein etabliertes therapeutisches Vorgehen bei TBA. Aufgrund der geringen Fallzahlen ist dazu bisher keine randomisierte Studie durchgeführt worden. Die Behandlung bei primärer TBA erfolgt hauptsächlich symptombezogen. Die Therapieentscheidungen müssen somit auf Einzelfallbasis getroffen werden. Bei asymptomatischen Patienten ist keine Therapie erforderlich, sie müssen lediglich nachbeobachtet werden, so wie auch im Fall unserer Patientin in den 10 Jahren seit der Diagnosestellung. Bei symptomatischen Patienten stehen die bronchoskopische Rekanalisierung, die medikamentöse Therapie und/oder die externe Strahlentherapie als Behandlungsoptionen zur Verfügung. Dadurch, dass die Amyloidläsionen so spröde sind, kommt es bei Eingriffen in aller Regel zu Blutungen. In der von Díaz-Jiménez et al. [7] beschriebenen Serie musste bei 2 von 11 Patienten die Resektion wegen exzessiver Hämorrhagie abgebrochen werden. Auch die medikamentöse Behandlung lokalisierter Amyloidosen stützt sich lediglich auf Fallberichtsstudien, formelle randomisierte kontrollierte Studien liegen nicht vor. Colchicin ist in einigen Fällen von TBA getestet worden, mit unterschiedlichen Ergebnissen [5, 10, 14]. Eine französische Studie beschreibt die Anwendung von Colchicin bei einem Patienten und berichtet von lokaler Streuung nach 7 Jahren Nachbeobachtung [14]. In einer chinesischen Fallserie wurden 6 von 64 Patienten mit Colchicin behandelt, jedoch liegen keine Berichte über die Nachbeobachtung vor [5]. Eine randomisierte Studie zur systemischen Amyloidose, in der Colchicin mit Melphalan oder Kortikoiden oder einer Kombination aller 3 Therapien verglichen wurde, ergab eine Senkung der Mortalität unter den Therapieregimen mit Melphalan und Kortikoiden [16]. Aufgrund der unterschiedlichen Pathogenese von systemischer und lokaler Amyloidose lassen sich diese Ergebnisse jedoch nicht auf die TBA-Therapie übertragen [16] Colchicin ist ein Inhibitor der mit dem familiären Mittelmeerfieber assoziierten Amyloidose sowie der Amyloidose im Mausmodell $[17,18]$. Es hemmt das Mikrotubulussystem in der Metaphase und bremst die Chemotaxe von Monozyten und Neutrophilen [18]. Colchicin kann die Ablagerung von Amyloidfibrillen in Organen hemmen, wie im Mausmodell nachgewiesen wurde [17]; dies ist möglicherweise auf seine entzündungshemmende Wirkung zurückzuführen. Andere Fallberichte zu TBA schließen eine Behandlung allein mit Glukokortikoiden oder in Kombination mit Colchicin ein und beschreiben einen allgemeinen Therapienutzen in einem Drittel der Fälle [5]. Unsere Patientin sprach sehr gut auf Colchicin als Monotherapie an, die subglottische Stenose klang vollständig ab und die tracheobronchiale Erkrankung besserte sich erheblich, wobei dies jedoch nicht zwingend nur der medikamentösen Therapie zugeschrieben werden kann. Die Amyloidablagerungen sind nicht statisch; es ist von ihrem Umsatz berichtet worden, und sie können sich bisweilen zurückbilden, wenn das Angebot an Vorläuferproteinen für die Fibrillen herabgesetzt ist [19]. Es ist auch nicht auszuschließen, dass die Krankheit spontan abgeklungen ist, wie schon einmal für einen einzelnen Fall beschrieben worden ist [20]. Wir halten diese letztere Hypothese angesichts des Ausmaßes der Erkrankung und der geringen Zahl berichteter Fälle in der Literatur jedoch für äußerst unwahrscheinlich.

Eine weitere Therapieoption ist die bronchoskopische Rekanalisierung der Atemwege. Hierzu sind bisher die Lasertherapie, mechanisches Debulking, die Ballondilatation und/oder die Stentimplantation beschrieben worden [5, 21, 22]. In einer chinesischen Fallserie mit 64 Patienten war bei 75\% der Patienten eine Therapie erforderlich; am häufigsten wurden die Nd:YAG-Lasertherapie und das mechanische Debulking gewählt. Teilweise wurde zwar über eine kurzfristige Linderung der Beschwerden berichtet, bei der Hälfte der Patienten war das Ansprechen jedoch schlecht [5]. Bei symptomatischer Kehlkopfamyloidose wird in der Regel ein endoskopischer Behandlungsansatz gewählt [1]; hier liegen über die Behandlung mit dem Kohlendioxid $\left(\mathrm{CO}_{2}\right)$-Laser und dem Nd:YAG-Laser die meisten Berichte vor [21, 23]. Auch externe Strahlentherapien sind mit guten Ergebnissen eingesetzt worden, allerdings ist der genaue Mechanismus, über den die positiven Effekte erzielt werden, ungeklärt und Evidenz liegt nur in Form von Fall-Kontroll-Studien vor [12, 23, 24]. Eine Ösophagitis stellt die häufigste Komplikation dar. Rückfälle im Zeitraum von 8 bis 80 Monaten nach der Behandlung sind berichtet worden [23]. Alle genannten Therapien sind nicht exklusiv, und in einigen Fällen wurde ein mehrgleisiger Therapieansatz versucht [23, 24]. Bei unserer Patientin waren angesichts ihres hervorragenden Ansprechens auf das Colchicin keine weiteren Behandlungsmaßnahmen erforderlich. Von der proximal-trachealen und subglottischen Erkrankung, die bei ihr vorlag, ist ebenso wie von der diffusen Form der TBA bekannt, dass sie mit einer schlechteren Prognose assoziiert sind $[10,13]$; dennoch ist die Patientin seit 4 Jahren durchgängig symptomfrei.

Zusammenfassend beschreiben wir den Fall einer Patientin mit langfristig dokumentierter Regression ad integrum ihrer TBA infolge einer Behandlung mit Colchicin. Unseres Wissens ist dies der erste Fallbericht über ein so gutes Ansprechen.

\section{Finanzielle Transparenz und Interessenkonflikte}

Die Autoren erklären, dass beim Verfassen dieses Artikels keine Interessenkonflikte vorlagen. 
1 Gillmore JD, Hawkins PN: Amyloidosis and the respiratory tract. Thorax 1999;54:444-451.

$\checkmark 2$ Glenner GG, Eanes ED, Page DL: The relation of the properties of Congo red-stained amyloid fibrils to the $\beta$-conformation. J Histochem Cytochem 1972;20:821-826.

-3 Utz JP, Swensen SJ, Gertz MA: Pulmonary amyloidosis. The Mayo Clinic experience from 1980-1993. Ann Intern Med 1996;124:407-413.

$\checkmark 4$ Capizzi SA, Betancourt E, Prakash UB: Tracheobronchial amyloidosis. Mayo Clin Proc 2000;75:1148-1152.

5 Ding L, Li W, Wang K, et al: Primary tracheobronchial amyloidosis in China: analysis of 64 cases and a review of literature. J Huazhong Univ Sci Technolog Med Sci 2010;30:599-603.

6 Hui AN, Koss MN, Hochholzer L, Wehunt WD: Amyloidosis presenting in the lower respiratory tract. Clinicopathologic, radiologic, immunohistochemical, and histochemical studies on 48 cases. Arch Pathol Lab Med 1986;110:212-218.

7 Díaz-Jiménez J, Rodríguez A, Martínez-Ballarín J, Castro MJ, Argemi T, Manresa F: Diffuse tracheobronchial amyloidosis. J Bronchology 1999;6:13-17.

8 Berk J, O’Regan A, Skinner M: Pulmonary and tracheobronchial amyloidosis. Semin Respir Crit Care Med 2002;23:155-165.

9 Borie R, Danel C, Molinier-Frenkel V, Prevot G, Deslee G, Debray MP, et al: Tracheobronchial amyloidosis: evidence for local B-cell clonal expansion. Eur Respir J 2012;39:1042-1045.
10 O’Regan A, Fenlon H, Beamis J, Steele M, Skinner M, Berk J: Tracheobronchial amyloidosis. The Boston University experience from 1984 to 1999. Medicine 2000;79:69-79.

11 Prowse CB: Amyloidosis of the lower respiratory tract. Thorax 1958;13:308-320.

12 Newton R, Kemp S, Yang G, Darzi A, Sheppard M, Shah P: Tracheobronchial amyloidosis and confocal endomicroscopy. Respiration 2011; 82:209-211.

13 Sommer P, Kumar G, Lipchik RJ, Patel JJ: Tracheobronchial amyloidosis managed with multimodality therapies. Ther Adv Respir Dis 2014;8:48-52.

14 Paccalin M, Hachulla E, Cazalet C, Tricot L, Carreiro M, Rubi M, et al: Localized amyloidosis: a survey of 35 French cases. Amyloid 2005; 12:239-245.

15 Thompson LD, Derringer GA, Wenig BM: Amyloidosis of the larynx: a clinicopathologic study of 11 cases. Mod Pathol 2000;13:528-535.

16 Kyle RA, Gertz MA, Greipp PR, Witzig TE, Lust JA, Lacy MQ, et al: A trial of three regimens for primary amyloidosis: colchicine alone, melphalan and prednisone, and melphalan, prednisone, and colchicine. N Engl J Med 1997;336:1202-1207.

17 Shtrasburg S, Pras M, Gal R, Salai M, Livneh A: Inhibition of the second phase of amyloidogenesis in a mouse model by a single-dose colchicine regimen. J Lab Clin Med 2001;138: 107-111.
Kucuk A, Gezer IA, Ucar R, Karahan AY: Familial Mediterranean Fever. Acta Medica (Hradec Kralove) 2014;57:97-104.

19 Hawkins PN: Studies with radiolabelled serum amyloid P component provide evidence for turnover and regression of amyloid deposits in vivo. Clin Sci 1994;87:289-295.

20 Hof DG, Rasp FL: Spontaneous regression of diffuse tracheobronchial amyloidosis. Chest 1979;76:237-239.

21 Alloubi I, Thumerel M, Begueret H, Baste J, Velly J, Jougon J: Outcomes after bronchoscopic procedures for primary tracheobronchial amyloidosis: retrospective study of 6 cases. Pulm Med 2012;2012:1-4.

22 Yang S, Chia SY, Chuah KL, Eng P: Tracheobronchial amyloidosis treated with rigid bronchoscopy and stenting. Surg Endosc 2003;17: 658-659.

23 Truong M, Kachnic L, Grillone G, et al: Longterm results of conformal radiotherapy for progressive airway amyloidosis. Int J Radiat Oncol Biol Phys 2012;83:734-739.

24 Neben-Wittich MA, Foote RL, Kalra S: External beam radiation therapy for tracheobronchial amyloidosis. Chest 2007;132:262-267. 